Boyd, J. S. K. \& Bidwet.L, D. E. (1959). J. gen. Microbiol. 21, 635-651

\title{
The Q1 (A) Strains of Salmonella typhimurium: Induction Phenomena
}

\author{
BY J. S. K. BOYD AND D. E. BIDWELL \\ The Wellcome Laboratories of Tropical Medicine, London, N.W. 1
}

\begin{abstract}
SUMMARY: Members of the Q1 (A) group of lysogenic strains of Salmonella typhimurium have a common bacterial component and differ only in their prophage content. All are inducible by ultraviolet (u.v.) radiation but, in the cultural conditions which were standard in the experiments here described, never more than $40 \%$ of the bacteria were induced, and this only with exposures which killed approximately $75 \%$ of the host strain, Q 1 . The latent period between irradiation and phage liberation was approximately $45 \mathrm{~min}$. The number of particles per burst varied widely. The most striking difference in the reaction to irradiation of cultures of the different strains was that, without significant variation in the amount of free phage produced, some showed clearing while others did not. Clearing was not directly related to the percentage of bacteria induced, nor was it attributable to the production of a lysin by some strains and not by others. Clearing occurred in strains in which induction took place in bacteria which would otherwise have survived irradiation, and would have enlarged into filamentous forms. In strains in which no clearing occurred, induction took place in bacteria which had received a lethal dose of u.v. radiation, and not in the more lightly irradiated bacteria which were therefore left free to enlarge and so mask the lysis of the induced bacteria. Thus there is evidence that the induction of these closely related prophages is 'triggered off' by different stimuli directly related to the degree of damage to the host bacterium caused by irradiation.
\end{abstract}

Lwoff, Siminovitch \& Kjeldgaard (1950) found that when lysogenic Bacillus megaterium was exposed to ultraviolet (u.v.) radiation under certain defined conditions it was 'induced': the innocuous prophage assumed virulent characters and multiplied at the expense of the host bacterium, producing a brood of free phage particles. Subsequent work has shown that many lysogenic bacteria of different species can be induced in this way, both by u.v. radiation and by other mutagenic agents. There is, however, considerable variation in the degree of induction produced in different bacteria and even in lysogenic bacteria of the same species (Gorrill \& Gray, 1956).

Recently, a series of twelve lysogenic strains of Salmonella typhimurium was prepared by infecting an indicator strain, Q1, with the different type $A$ phages which have been recovered from wild strains of that organism (Boyd \& Bidwell, 1957). This will be referred to as the Q1 (A) series. In all members of the series the bacterial component is the same: only the prophages differ. Thus any variation in reaction which these lysogenic strains show must be attributed to the prophage. A few preliminary experiments showed that there was in fact a very considerable variation in response to u.v. radiation, and it was deemed of interest to explore this phenomenon more carefully. It must be re-emphasized that these phages are not artificially produced mutants of a 
common ancestor. They have all been recovered, in many cases repeatedly: from wild strains of $S$. typhimurium from cases of food poisoning or from carriers.

\section{METHODS}

Two indicator strains were used, Salmonella typhimurium $\mathrm{Q}$, which is sensitive to all the A phages, and $S$. gallinarum SL 140, a streptomycin-resistant strain kindly provided by Dr B. Stocker. $S$. gallinarum is an efficient indicator for a wide range of Salmonella phages.

The culture media were those detailed previously (Boyd, 1950) and the buffer that of Visconti \& Delbrück (1953). During incubation, cultures were neither aerated nor shaken, the active movements of the bacteria being deemed adequate to ensure collisions between them and the phage particles.

The optical density of the cultures from which growth curves were plotted was estimated by the EEL nephelometer; this instrument proved very suitable for the purpose, as readings could be made quickly and with little exposure of the culture tubes to light, so avoiding the risks of photorestoration.

For u.v. radiation a Philips Germicidal Lamp of $30 \mathrm{~W}$. was used at a distance of $1 \mathrm{~m}$. from the cultures, the lamp being allowed to heat up for $15 \mathrm{~min}$. before being used. The efficacy of the irradiation was estimated by preliminary tests which will be described. This work has been in progress for some years. In earlier experiments there were certain unsuspected factors which affected the length of exposure. It has been possible to make accurate adjustments; and the corrected results of these earlier experiments have been embodied with those of later experiments. The exposures quoted throughout the paper are in terms of the later experiments.

To prepare bacteria for irradiation tubes of broth $(10 \mathrm{ml}$.) were inoculated from overnight agar slopes, incubated for $3 \mathrm{hr}$. at $37^{\circ}$, and then centrifuged. The bacteria were resuspended in buffer of equal volume to the original cultures, and the contents of the tubes pooled. Two ml. of this was added to $8 \mathrm{ml}$. of broth for control purposes. Prepared in the above way, this control contains approximately $10^{8}$ viable bacteria/ml. The remainder was placed in quantities of $3 \mathrm{ml}$. in $10 \mathrm{~cm}$. Petri dishes, as many as required for the particular experiment. Each sample for irradiation was placed on a rocking platform at the appropriate distance from the lamp and the exposure was made by removing and replacing the lid of the Petri dish like the lens cap of a studio camera. After irradiation, $2 \mathrm{ml}$. of the suspension was added to $8 \mathrm{ml}$. of broth, giving the same concentration of bacteria as in the control. Incubation was carried out at $37^{\circ}$ in a Grant water bath with a metal cover to exclude light.

The percentage of bacteria surviving exposure to u.v. radiation was estimated by making viable counts before and after irradiation. The percentage of bursts, i.e. of bacteria which undergo induction and lysis after irradiation, was found by diluting the irradiated culture to give approximately one organism/0.4 ml., and charging each of 100 sterile tubes $\left(4\right.$ in. $\times \frac{1}{2}$. in. $)$ with this 
volume. To ensure that all induced bacteria burst, the tubes were incubated in the water bath for $2 \mathrm{hr}$. at $37^{\circ}$. Thereafter, to save unnecessary manipulation, a preliminary test was made by spotting a loopful from each tube on numbered sections of a plate of agar freshly sown with the indicator strain (a 'lawn'). After overnight incubation, the tubes in which bursts had occurred were identified by the presence of plaques in the numbered spots, and an accurate count of the phage particles in each of these tubes was made by standard methods to calculate the number of particles produced in each burst. As it is impossible to distribute the bacteria in the tubes with mathematical accuracy (i.e. when 100 bacteria are shared into 100 tubes with the theoretical distribution of 1 bacterium/tube, approximately one-third of the tubes are found to contain no bacteria, and certain of the remaining tubes 2,3 and even 4 bacteria) a correction factor based on the Poisson distribution was used to convert 'tubes showing bursts' to 'estimated numbers of induced bacteria'. A few confirmatory experiments were carried out using a dilution estimated to give one bacterium/4 tubes: these gave comparable results.

No attempt was made, either by varying the medium or by other means, to increase the yield of phage particles in an irradiated culture.

Smears for microscopical examination were dried and fixed, either by methyl alcohol to be stained by Giemsa's stain, or by heat to be stained by borax methylene blue or carbol fuchsin. Special methods of staining designed to show internal structure were not employed.

\section{RESULTS}

Before proceeding to consider the effects of u.v. radiation on the $Q 1$ (A) series, some recent observations on $\mathrm{Q} 1$, the parent strain, must be recorded. This strain was derived from a wild Salmonella typhimurium, carrying prophage A3, which was isolated from the faeces of a patient suffering from food poisoning. During a prophage substitution experiment a few bacteria in the cultures were 'cured' of their A phage infection, and a colony which grew from one of these cured organisms was the origin of the Q1 strain. At first, Q1 was thought to be phage-free, as no phage arising from it could be detected on the indicator strains 1404 and 1411 used at that time. However, Dr B. Stocker has recently found (personal communication) that Q1 contains a phage for which $S$. gallinarum strain SL 140 is an indicator-an observation which was readily confirmed. On a lawn of SL 140, the phage from Q1 produces small punched-out plaques, about $0 \cdot 1-0 \cdot 15 \mathrm{~mm}$. in diameter, which under high magnification are seen to have a minute central spot of bacterial growth. This phage is neutralized by the antiserum of phage B5 (Boyd, 1950), and further resembles B5 by showing considerable heat resistance: a limited number of particles survive exposure for $30 \mathrm{~min}$. to $\mathbf{7 0 ^ { \circ }}$, but all are destroyed by heating for $30 \mathrm{~min}$. at $75^{\circ}$. It differs from $\mathrm{B} 5$, of course, in its host range. This phage from Q1 has been designated B 5d.

Phage lysates, prepared from the Q1 (A) series, were spotted on a lawn of Salmonella gallinarum strain SL 140. This revealed in every case plaques of 
the A phage and of phage B 5d. All these strains are therefore doubly lysogenic, carrying both their particular A phage and B 5 d.

When cultures of the $Q 1$ (A) series are induced by u.v. radiation, it is found that the A phage take priority over B 5d. The two phages are induced independently. On rare occasions both phages have been found in the same tube in the burst experiments to be detailed later, but no more often than could be accounted for by the occasional presence of two bacteria instead of one in the tube. The percentage of B $\mathrm{d}$ bursts is much lower in irradiated Q1 (A) cultures than in irradiated Q1, and for some reason, for which no explanation can be offered, the number of $\mathrm{B} 5 \mathrm{~d}$ phage particles released per burst is also much lower in irradiated Q1 (A) strains than in irradiated Q1.

In the experiments to be recorded, the presence of phage B 5d in strain $Q 1$ has no particular significance, and to avoid confusion no reference will be made to it, but as a matter of interest, the B 5d figures are embodied in Fig. 10.

Irradiation of the parent strain Salmonella typhimurium $Q 1$

Morphological changes resulting from irradiation. Stained smears were prepared at intervals from an irradiated culture of Salmonella typhimurium Q 1 subsequently incubated at $37^{\circ}$. Immediately after irradiation and before incubation, numerous organisms were seen in various stages of division. After incubation for $15 \mathrm{~min}$. dividing forms were fewer in number and after $\mathbf{3 0} \mathrm{min}$. they were scanty. After 45 min. many organisms were seen to be swollen and elongated, and a few, about twice the normal size, were boat-shaped, with deeply stained ends and a clear centre. The process of enlargement continued up to 90 min., when numerous filamentous bacteria measuring 11-20 $\mu$ were present. Subsequently, these filaments grew proportionately fewer in number, while small dividing forms reappeared. Some of the filaments stained irregularly, others were beaded, and some fragmented, giving a streptobacillus-like appearance. After overnight incubation of the irradiated broth cultures, occasional filaments were still to be seen and, in general, the bacteria showed much irregularity in size, the average being greater than that of the organisms in the original culture. The fate of the filamentous forms was followed by keeping a live preparation under continuous observation with phase-contrast illumination, using a technique previously described (Boyd, 1949). A minority were found to remain in this enlarged state indefinitely. The majority divided after $90 \mathrm{~min}$. or more of incubation from the time of irradiation, and the daughters continued to divide. Those of the first generation were from 2 to 3 times the size of normal bacteria, those of the second generation were still enlarged, though not so big as their parents, and those of subsequent generations were approximately normal in size. Thus the majority of the filamentous forms were not moribund as might at first be supposed, but in time divided and produced apparently normal progeny. (A similar observation was made in the case of Escherichia coli by Deering \& Setlov, 1957.) Only a minority of the bacteria which became enlarged and swollen failed to survive. From this it may be deduced that the majority of the bacteria which were killed by irradia- 
tion (apart from the action of induction) were affected to such an extent that they did not increase in size but were, as it were, killed outright.

Lethal effect of u.v. radiation. Strain Q1, after the standard preparatory treatment, was irradiated for varying periods, and the lethal effect of this treatment was estimated by counting the viable bacteria in samples taken before and after irradiation. The findings in repeated experiments of the kind were remarkably consistent, though it was noted that minor variations occurred which were related to the concentration of the bacteria in the suspension at the time of irradiation. Thus, with a denser suspension the percentage of surviving bacteria was higher, a result probably attributable to 'shadowing'. The results are shown in Table 1.

Table 1. Lethal effect of ultra-violet radiation on Salmonella typhimurium strains Q1, Q1 $(A 1 b)$ and $Q 1(A 1 c)$

Suspension of Salmonella typhimurium, strains Q1, Q1 (A 1b) and Q1 (A 1c), were prepared for irradiation by the methods described. The viable bacteria were counted (1) in the suspensions immediately before irradiation, and (2) in portions of the suspensions immediately after exposure to u.v. radiation for the times indicated. The 'survivors' are shown as a percentage of the viable bacteria in the suspension before irradiation. The figures given for exposures of 5 and 10 sec. are the mean of several experiments.

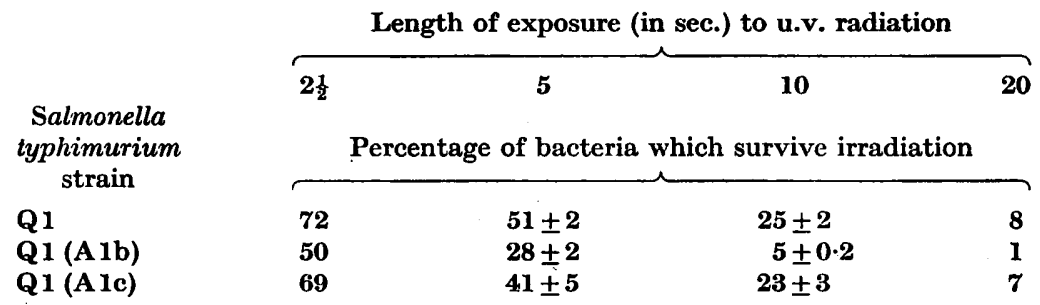

Effect of u.v. radiation on turbidity curves. The process of growth with arrested division is reflected in the turbidity curves plotted in Fig. 1. The preliminary increase in opacity, which occurred in the first $90 \mathrm{~min}$. of incubation, resulted from the enlargement of the bacteria and not, as in the control, from multiplication. The flattening of the curve which is seen for the next 30-60 min. cannot be attributed to lysis resulting from the induction of phage B 5d, for this lysis, as shown by the rising titre of free phage, occurred between 45 and 60 min. after irradiation, at which time the turbidity curve was still rising: it is evidence of the persisting effect of irradiation on the metabolism of the surviving bacteria, causing a slowing down of their growth rate.

It is noteworthy that the more lightly irradiated cultures showed a greater increase in opacity during the first $90 \mathrm{~min}$. of incubation than did those which were more heavily irradiated, a fact which suggests that minor damage interfered with division without greatly influencing growth, whereas more severe damage brought both division and growth to an end by killing the organism. 


\section{Ultraviolet radiation of free particles of type $A$ phages}

Latarjet, Morenne \& Berger (1953) have shown that coliphage $\mathrm{T}_{2}$ is quickly destroyed by irradiation. Both temperate and virulent $\mathbf{A}$ phages are much more resistant. The survival rates of coliphage $T_{2}$ and Salmonella phage $A \mathbf{~} \mathbf{b}$ are compared in Fig. 2. The other phages of the $\mathbf{Q} \mathbf{1}(\mathrm{A})$ series are similarly resistant, and the exposure of the lysogenic cultures to irradiation for $20 \mathrm{sec}$. (the longest time adopted in most of the experiments which follow) produced only a negligible decrease in the number of free phage particles when counts were made immediately after exposure. It is of interest to recall that these same phages are very resistant to heat, and survive exposure to a temperature of $80^{\circ}$ for $30 \mathrm{~min}$.

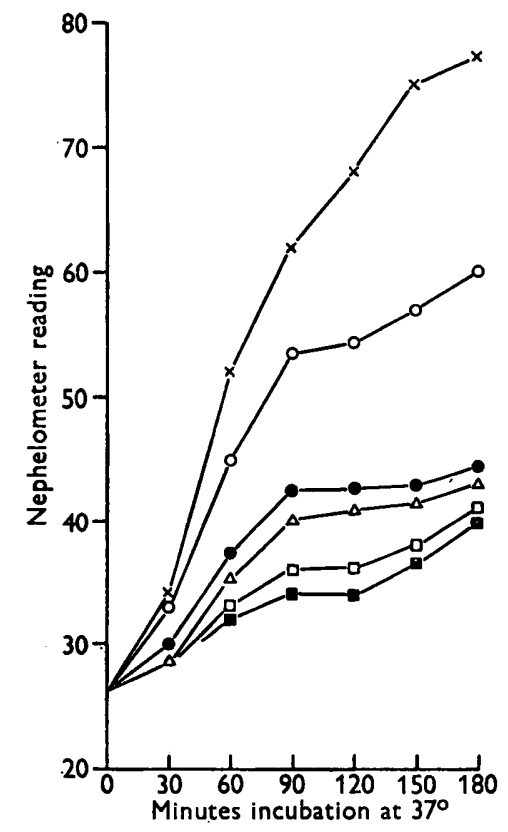

Fig. 1

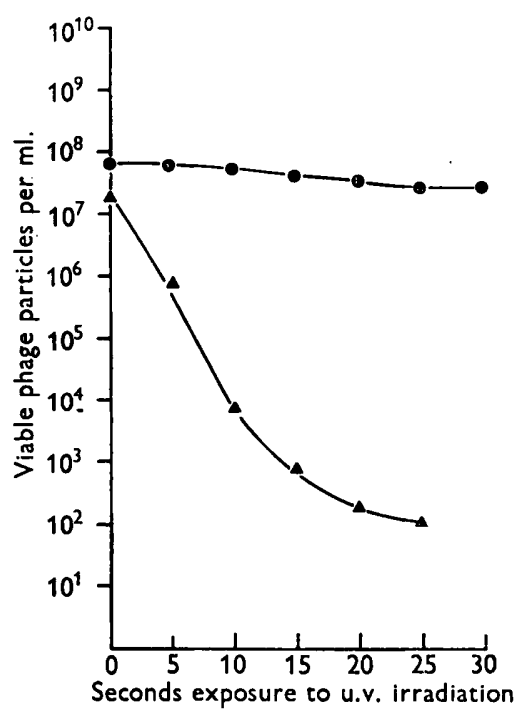

Fig. 2

Fig. 1. Turbidity curves of incubated cultures of Salmonella typhimurium, strain Q1, after varying exposures to $u$.v. radiation. With increasing exposures the curves become lower and flatter, but show no abrupt fall indicating mass lysis. Seconds irradiation: $\times, 0$; $0,2.50 ; 0,5 \cdot 00 ; \triangle, 9 \cdot 5 ; \square, 10 \cdot 0 ; \square, 12 \cdot 5$.

Fig. 2. Lethal effect of $u$.v. radiation on free phage particles of coliphage $T_{2}$ and Salmonella phage A1b. $\Delta$, Bacteriophage $T_{2} ; 0$, bacteriophage Alb.

\section{Ultraviolet radiation of the $Q 1(A)$ series of Salmonella phages}

Preliminary tests. A series of preliminary observations was made by irradiating all 12 lysogenic strains for 5 sec. and thereafter incubating and recording turbidity at intervals for $3 \mathrm{hr}$. The resultant curves varied considerably. In two there was evidence of well-marked lysis, i.e. almost complete clearing of the culture; in others there was less obvious lysis, while in the remainder there 
was retardation of growth of varying degrees with no fall in the curve to suggest mass lysis.

The 12 strains were then irradiated for 10 sec., and the titre of free phage particles estimated after incubation for $90 \mathrm{~min}$. The results, together with an assessment of the degree of clearing which occurs, are shown in Table 2. It will be seen that while all 12 strains were induced, there was some variation in the final concentration of phage. This however bore no constant relationship to the degree of clearing. The highest titre was found in the culture of Q1 (A2d), which showed well-marked clearing, but other types which showed no clearing, such as Q1 (A 1c), had almost as high a titre as Q1 (A 1b), in which clearing was marked.

Table 2. Comparison of results of irradiation of the $Q 1(A)$ series of Salmonella typhimurium

Bacterial lysis was estimated by nephelometer readings of broth cultures taken at intervals during incubation at $37^{\circ}$. ++ denotes the degree of clearing seen in Fig. 3 ; - denotes absence of clearing as seen in Figs. 1 and $4 ;+$ and \pm denote intermediate degrees. Suspensions were prepared for irradiation by the methods described, and the free phage particles in these suspensions were counted before exposure to u.v. radiation. Portions of each suspension were irradiated for 5 sec., and thereafter incubated together with unirradiated suspensions as controls. The free phage particles were counted after incubating for 90 min. The control shows the normal increase in free phage which is found in an actively growing lysogenic culture. The much higher count in the irradiated sample results from induction of a proportion of the lysogenic bacteria.

\begin{tabular}{|c|c|c|c|c|}
\hline & $\begin{array}{c}\text { Degree of } \\
\text { bacterial } \\
\text { lysis }\end{array}$ & $\begin{array}{c}\text { Phage count } \\
\text { before } \\
\text { irradiation }\end{array}$ & $\begin{array}{l}\text { Phage count } \\
\text { in control } \\
\text { culture } \\
\text { incubated } \\
90 \text { min. }\end{array}$ & $\begin{array}{c}\text { Phage } \\
\text { count in } \\
\text { irradiated } \\
\text { culture } \\
\text { incubated } \\
90 \text { min. }\end{array}$ \\
\hline Q1 (A 1a) & + & $5.2 \times 10^{4}$ & $1.8 \times 10^{6}$ & $9.0 \times 10^{8}$ \\
\hline (A1b) & ++ & $2.4 \times 10^{5}$ & $1.4 \times 10^{6}$ & $1.9 \times 10^{9}$ \\
\hline (A 1c) & - & $2.7 \times 10^{4}$ & $8.0 \times 10^{5}$ & $1.6 \times 10^{9}$ \\
\hline (A Id) & - & $2.3 \times 10^{4}$ & $5.6 \times 10^{5}$ & $2.7 \times 10^{9}$ \\
\hline (A 2a) & + & $1.8 \times 10^{5}$ & $1.0 \times 10^{6}$ & $3.8 \times 10^{9}$ \\
\hline (A2b) & \pm & $7 \cdot 2 \times 10^{3}$ & $1.3 \times 10^{5}$ & $2.3 \times 10^{9}$ \\
\hline (A 2c) & $\frac{1}{t}$ & $1.7 \times 10^{8}$ & $6.8 \times 10^{5}$ & $1.7 \times 10^{8}$ \\
\hline (A2d) & ++ & $3.9 \times 10^{5}$ & $1.0 \times 10^{6}$ & $5.2 \times 10^{9}$ \\
\hline (A2e) & + & $3.6 \times 10^{3}$ & $6.0 \times 10^{4}$ & $2.7 \times 10^{8}$ \\
\hline (A2f) & - & $4.8 \times 10^{3}$ & $7 \cdot 2 \times 10^{4}$ & $1.1 \times 10^{8}$ \\
\hline (A3) & - & $4.8 \times 10^{5}$ & $1.1 \times 10^{7}$ & $1.4 \times 10^{9}$ \\
\hline (A4) & - & $1.2 \times 10^{5}$ & $2.8 \times 10^{6}$ & $6.4 \times 10^{8}$ \\
\hline
\end{tabular}

Selection of strains $Q 1(A 1 b)$ and $Q 1(A 1 c)$ for detailed investigation. Two strains were selected for detailed investigation, namely Q1 (A1b) which showed well-marked clearing, and Q1 (A 1c), which showed retardation of growth but no clearing. A third strain, Q1 (A 1a), which showed a limited degree of clearing, was also examined in the same detail; the results of the various investigations carried out on this strain lay so consistently in an intermediate position between those of the other two, that nothing is to be gained by recording them.

Prophages A 1b and Alc confer a high degree of immunity on their host bacteria. Not only do strains Q1 (A 1b) and Q1 (A 1c) show mutual cross- 
immunity to the phages they produce, but they are also, with minor exceptions, resistant to all the $\mathbf{A}$ phages (Boyd \& Bidwell, 1957). Conversely, phages A 1 b and A 1c multiply freely on 6 of the 12 strains of the Q1 (A) series. There are, however, some minor differences in the cross-immunity reactions of the two strains by which they can be identified; they also show variations in plaque characters and in host range. Nevertheless, they appear to be more closely related than any other members of the $\mathbf{A}$ series, and it is therefore the more interesting that they should differ so widely in their reactions to u.v. radiation. Prophage Alb is the phage of Lilleengen's LT22 strain 409 (Lilleengen, 1948) used by Zinder \& Lederberg (1952) in their transduction experiments.

Morphological changes after irradiation. On incubation at $37^{\circ}$, the morphological characters of the bacteria in irradiated cultures of these lysogenic strains differ notably. Strain Q1 (A 1c), which has growth curves resembling those of strain $Q 1$, but slightly flatter, undergoes changes very similar to those shown by $Q 1$, which have already been described. Division is arrested, and many of the organisms become grossly enlarged and filamentous. After incubation for $90 \mathrm{~min}$. these enlarged forms become progressively fewer, but some are still present in an overnight culture.

Strain Q1 (A 1b), which shows well-marked clearing, presents a different picture. In the first $\mathbf{4 5} \mathrm{min}$. of incubation it develops in much the same way as Q1, except that a larger percentage of the organisms have a boat-like appearance, with polar staining. From this stage onwards, progress is very different. The number of bacteria in the smear notably decreases; only a few filamentous forms, so conspicuous a feature in corresponding smears of $Q 1$ and Q1 (A 1c), develop; the boat-like organisms disappear, and most of the remaining bacteria are of normal size.

Turbidity curves. Cultures of strains Q 1 (A 1 b) and Q 1 (A 1c) were irradiated and incubated. The turbidity of the cultures was recorded at $30 \mathrm{~min}$. intervals, and the curves plotted (Figs. 3, 4). These conform to the findings of the preliminary experiments. In Q1 (A Ib) well-marked clearing occurred after incubation for about $90 \mathrm{~min}$. The general pattern of the curve was not changed by increased exposure, the only significant difference being that the cultures which received the longer exposures had lower turbidity readings. The curves of Q1 (A 1c) are much like those of Q1 (Fig. 1), but run at a lower level. No noticeable clearing occurred, though the curves were flattened.

Counts of viable bacteria in cultures immediately after irradiation. The lethal action of u.v. radiation on strains Q1, Q1 (A 1b) and Q1 (A 1c) is shown in Table 1. Q1 (A 1c) showed a slightly lower survival ratio than Q1; in Q1 (A 1 b) the survivors were markedly fewer than in the first two.

Counts of viable bacteria in irradiated cultures after different periods of incubation. It seemed possible that the clearing which occurred in an irradiated culture of strain $Q 1$ (A lb) might have resulted from the action of a lysin liberated from the induced bacteria. To test this hypothesis, cultures of Q1 (A 1c) and Q1 (A 1b), prepared in the standard way, were irradiated for 5 sec. and incubated in parallel with an unirradiated control. Counts of the 
viable bacteria in both preparations were made immediately after irradiation. It was assumed that the lysin was unlikely to function unless it was present in a concentrated form. Accordingly, viable counts were made at intervals (1) from the original irradiated culture containing approximately $10^{8}$ bacteria (living or dead)/ml., and (2) from secondary cultures prepared by diluting the original $0.2 \times 10^{-5}$, giving a content of $2 \times 10^{2}$ bacteria (living or dead)/ml., and a correspondingly decreased concentration of any lysin which might be present. The results are shown in Figs. 5 and 6 . The turbidity curves of controls and irradiated cultures follow the usual pattern. The viable bacteria are plotted as a percentage of the number originally present in the unirradiated

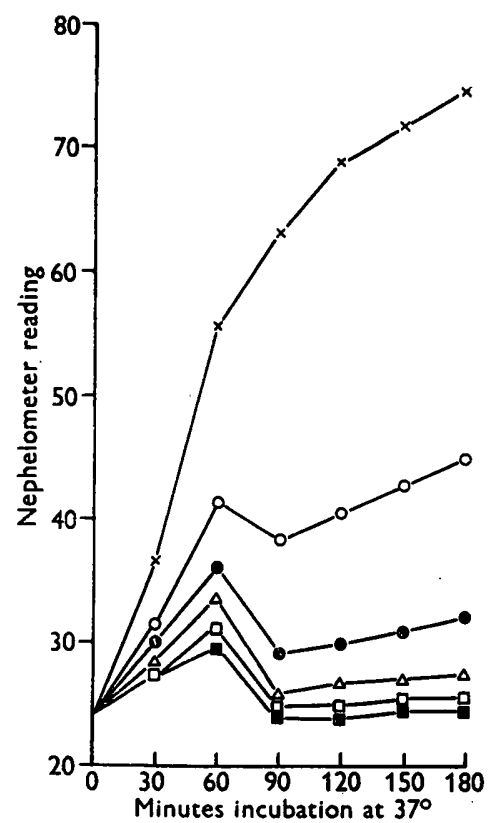

Fig. 3

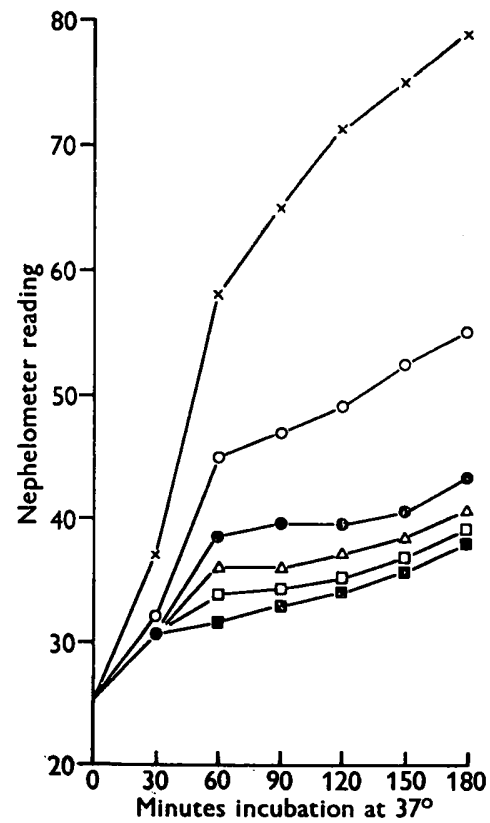

Fig. 4

Fig. 3. Turbidity curves of incubated cultures of $S$. typhimurium, strain $\mathbf{Q} 1$ (A 1b), after varying exposures to $u . v$. radiation. After incubation for $60 \mathrm{~min}$. clearing occurs at all exposures. Seconds irradiation: $x, 0 ; 0,2 \cdot 5,0,5 ; \Delta, 7 \cdot 5 ; \square, 10 ; \square, 12.5$.

Fig. 4. Turbidity curves of incubated cultures of $S$. typhimurium strain Q1 (A 1c) after varying exposures to $u . v$. radiation. The curves are similar to those of strain $Q 1$ (Fig. 1), but run at a lower level. Seconds irradiation: $x, 0 ; 0,2 \cdot 5 ; 0,5 ; \Delta, 7 \cdot 5$, $\square, 10 ; \square, 12.5$.

control. In both irradiated cultures the number of viable bacteria decreased during the first $60 \mathrm{~min}$. of incubation, but fairly rapid multiplication (which, as would be expected, was more marked in the diluted cultures containing fresh medium) set in after about $90 \mathrm{~min}$. The decrease in viable bacteria was greater in the undiluted cultures, indicating the presence of some lethal agent. But the difference between strain $Q 1(A 1 b)$, in which clearing occurred, and strain $Q 1$ (A 1c) in which there was no clearing, was not sufficient to be regarded as significant. 
Liberation of endolysin. Experiments were carried out along the lines suggested by Jacob \& Fuerst (1958). Cultures of strains Q 1 (A 1b) and Q 1 (A 1c), prepared by standard methods, were irradiated for 5 sec., and different samples were incubated for $50 \mathrm{~min}$. (the point at which clearing was about to begin in Q1 (A 1b)) and $120 \mathrm{~min}$ (when clearing was complete). These samples, both with and without the addition of Tween 80 (to counteract possible adsorption), were centrifuged and filtered through Gradocol membranes to remove the phage, and were then tested against cultures of strains Q1, Q1 (A 1b) and Q1 (A 1c) which had first been treated with ethylenediamine tetra-acetic acid. There was no detectable clearing, from which it would appear that no endolysin was liberated in the irradiated cultures.

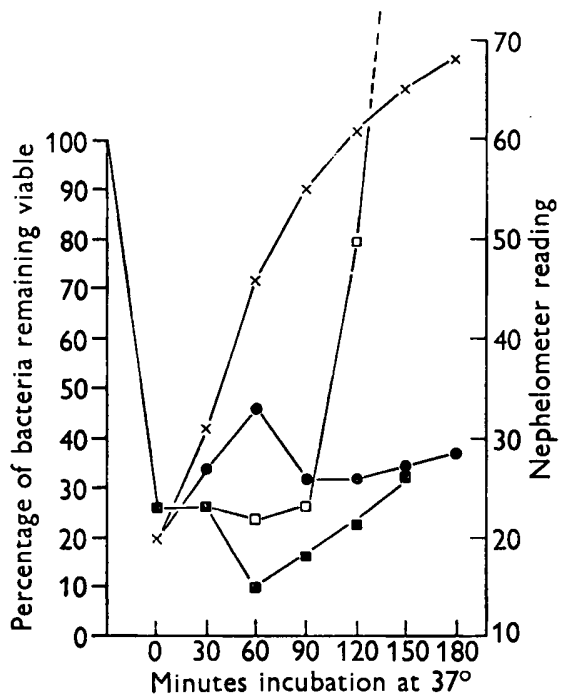

Fig. 5

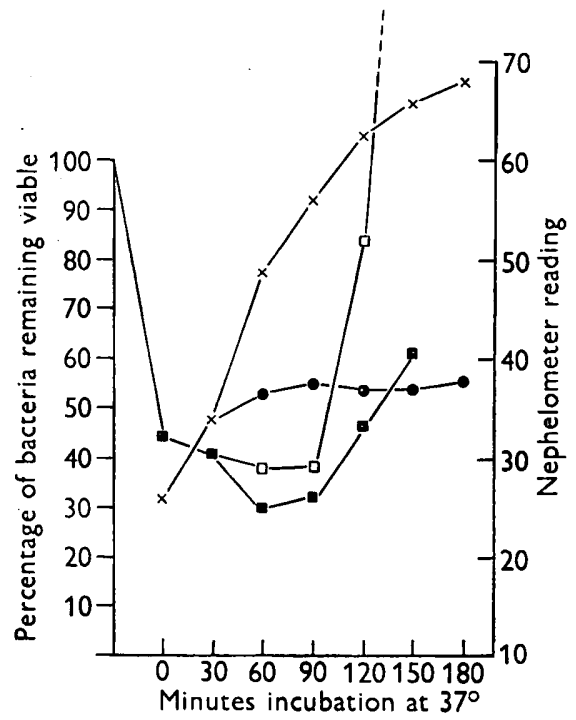

Fig. 6

Fig. 5. Progressive lethal action of u.v. radiation on $S$. typhimurium strain $Q 1$ (A 1b). In an undiluted culture the count of viable bacteria falls during the first 60 min. of incubation. A portion of the same culture diluted $0.2 \times 10^{5}$ shows no such fall. $\times$, Turbidity curve of control culture; $\ominus$, turbidity curve of irradiated culture; $\square$, count of viable bacteria made from diluted culture; $a$, count of viable bacteria made from undiluted culture.

Fig. 6. Progressive lethal action of u.v. radiation on $S$. typhimurium strain $Q 1$ (A 1c). The fall in the viable count is slightly less than in strain $Q 1$ (A 1b) (Fig. 5). $\times$, Turbidity curve of control culture; $O$, turbidity curve of irradiated culture; $\square$, count of viable bacteria made from diluted culture; $\square$, count of viable bacteria made from undiluted culture.

Influence of length of exposure on induction as evidenced by the liberation of free phage. To ascertain the effect of different lengths of exposure to u.v. radiation, a series of standard suspensions of the two lysogenic strains was irradiated for different periods, incubated for $90 \mathrm{~min}$. (the time at which the optimum phage titre was reached in an induced culture), diluted in streptomycin broth to kill the surviving bacteria, and counted on Salmonella gallinarum, strain SL 140. The results are plotted in Fig. 7. The maximum titre 
was reached after 5-10 sec. exposure. The number of particles liberated by the induction of strain $\mathrm{Q} 1$ ( $\mathrm{Alb}$ ) was higher than in the corresponding preparation of Q1 (A 1c), but not strikingly so. The titre of free phage has varied slightly in different experiments of this type, probably in relation to the bacterial count of the suspension, but the pattern of the opacity curve has been constant. On no occasion has Q1 (A 1c) shown detectable clearing.

Length of exposure and percentage of bacteria induced. Suspensions of strains Q1 (A 1b) and Q1 (A 1c), prepared by the standard technique, were irradiated for different lengths of time, and thereafter the number of bursts was determined by the method already described. In some cases repeated experiments were performed; the results shown in Table 3 are the mean of the findings. An exposure of 5 sec. gave the highest number of bursts. With exposures of $2 \frac{1}{2}$ sec. the number of bacteria induced in cultures of strain Q1 (A1b) was slightly higher than in the case of Q1 (A1c). With an exposure of $5 \mathrm{sec}$. induction was practically alike in both. With an exposure of $10 \mathrm{sec}$., the induction of Q1 (A Ic) was much decreased, being only about half of that of Q1 (A 1b). As an exposure of 5 sec. produced a well-marked clearing in subsequently incubated cultures of strain $Q 1(A 1 b)$, and none in $Q 1$ (A 1c) (see Figs. 3,4 ) it can be concluded that clearing is not to be explained solely in terms of the percentage of bacteria in a culture which are induced.

\section{Table 3. Influence of length of exposure to ultra-violet radiation on the percentage of bacteria induced}

Standard suspensions of bacteria were irradiated for the times shown. The suspensions were then diluted to give an average of 1 bacterium $/ 0.4 \mathrm{ml}$. and distributed in $0.4 \mathrm{ml}$. quantities in 100 small test tubes. In some experiments the dilution was greater, giving an average of 1 bacterium/1.6 ml. : this was also distributed in $0.4 \mathrm{ml}$. quantities in 200 small test tubes, giving an average of 1 bacterium/4 tubes. The tubes were incubated for $2 \mathrm{hr}$. and thereafter tested for the presence of free phage particles (indicating that the bacterium had been induced). A correction factor based on the Poisson distribution was used to convert 'tubes in which bursts occurred' to 'percentage of bacteria induced'; this is shown as the nearest whole number. Several experiments were carried out with exposures of 5 and $10 \mathrm{sec}$.

\begin{tabular}{|c|c|c|c|c|}
\hline & \multicolumn{4}{|c|}{ Length of exposure (in sec.) to u.v. radiation } \\
\hline Salmonella typhimurium & \multicolumn{4}{|c|}{$\begin{array}{c}\text { Percentage of bacteria which 'burst' as a result of } \\
\text { induction of the } A \text { prophage }\end{array}$} \\
\hline $\begin{array}{l}\text { Q1 (A 1b) } \\
\text { Q1 (A 1c) }\end{array}$ & $\begin{array}{l}29 \\
25\end{array}$ & $\begin{array}{l}36 \pm 5 \\
36 \pm 2\end{array}$ & $\begin{array}{l}35 \pm 3 \\
16 \pm 2\end{array}$ & $\begin{array}{r}24 \\
5 \cdot 0\end{array}$ \\
\hline
\end{tabular}

Number of phage particles produced by an induced bacterium. There was gross variation in the burst size, i.e. the number of phage particles produced by different bacteria when induced. The results of several experiments with Salmonella typhimurium are compiled in Table 4. In many cases the high figures at the upper end of the range are to be attributed to the presence of more than one induced bacterium/tube, but even when this is taken into account the spread is very wide. There has also been considerable variation in different experiments: for no obvious reason the burst size was much above the average 
Table 4. Range of 'burst' sizes

These counts were made on the 'bursts' recorded in Table 3. The striking feature is the wide scatter of burst sizes in every experiment, including those experiments in which the dilution was such that the chances of two induced bacteria being present in the same tube were remote.

\begin{tabular}{|c|c|c|c|c|c|c|}
\hline $\begin{array}{c}\text { Salmonella } \\
\text { typhimurium } \\
\text { strain }\end{array}$ & $\begin{array}{c}\text { Exposure } \\
\text { to u.v. } \\
\text { radia- } \\
\text { tion } \\
\text { (sec.) }\end{array}$ & $\begin{array}{l}\text { Viable bacteria } \\
\text { per ml. before } \\
\text { irradiation }\end{array}$ & $\begin{array}{c}\text { Theoretical } \\
\text { distribution } \\
\text { of bacteria } \\
\text { per tube }\end{array}$ & $\begin{array}{l}\text { No. of tubes } \\
\text { showing } \\
\text { bursts }\end{array}$ & $\begin{array}{l}\text { Lowest } \\
\text { no. of } \\
\text { particles }\end{array}$ & $\begin{array}{l}\text { Highest } \\
\text { no. of } \\
\text { particles }\end{array}$ \\
\hline \multirow[t]{8}{*}{ Q1 (A 1c) } & 5 & $1.0 \times 10^{8}$ & $1 / 1$ & $30 / 100$ & 18 & 420 \\
\hline & 5 & $1.2 \times 10^{8}$ & $1 \cdot 2 / 1$ & $38 / 100$ & 26 & 624 \\
\hline & $\mathbf{5}$ & $1 \cdot 14 \times 10^{8}$ & $0 \cdot 285 / 1$ & $19 / 200$ & 48 & 392 \\
\hline & 5 & $1.08 \times 10^{8}$ & $0 \cdot 27 / 1$ & $21 / 200$ & 54 & 384 \\
\hline & 10 & $1.1 \times 10^{8}$ & $1 \cdot 1 / 1$ & $13 / 100$ & 82 & 1308 \\
\hline & 10 & $1.03 \times 10^{8}$ & $1.03 / 1$ & $17 / 100$ & 38 & 536 \\
\hline & 10 & $0.8 \times 10^{8}$ & $0.8 / 1$ & $12 / 100$ & 80 & 598 \\
\hline & 10 & $1.07 \times 10^{8}$ & $1 \cdot 07 / 1$ & $15 / 100$ & 12 & 470 \\
\hline \multirow[t]{7}{*}{ Q1 (A lb) } & 5 & $1.16 \times 10^{8}$ & $1 \cdot 16 / 1$ & $38 / 100$ & 6 & 416 \\
\hline & 5 & $0.9 \times 10^{8}$ & $0.9 / 1$ & $33 / 100$ & 8 & 394 \\
\hline & 5 & $1.1 \times 10^{8}$ & $0 \cdot 275 / 1$ & $17 / 200$ & 22 & 322 \\
\hline & 5 & $1.08 \times 10^{8}$ & $0.27 / 1$ & $16 / 200$ & 16 & 232 \\
\hline & 10 & $0.84 \times 10^{8}$ & $0.84 / 1$ & $23 / 100$ & 8 & 600 \\
\hline & 10 & $0.9 \times 10^{8}$ & $0.9 / 1$ & $29 / 100$ & 32 & 300 \\
\hline & 10 & $1.04 \times 10^{8}$ & $0.04 / 1$ & $32 / 100$ & 12 & 398 \\
\hline
\end{tabular}

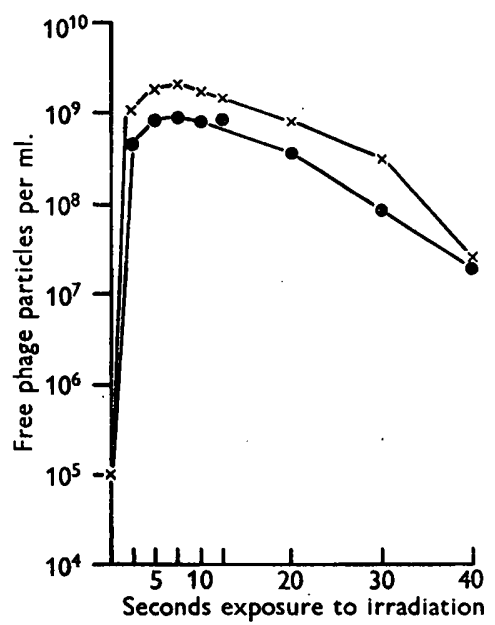

Fig. 7

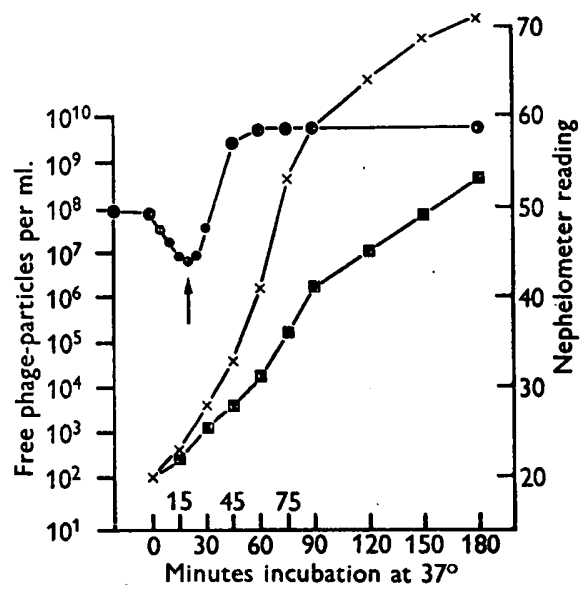

Fig. 8

Fig. 7 . The titre of free phage particles, resulting from the induction of S.typhimurium strains $Q 1$ (A 1 b) and Q1 (A 1c), in cultures exposed to ultraviolet radiation for varying periods. Optimum induction results from exposures of 5-10 sec. Strain Q1 (A 1b) gives a higher titre than Q1 (A 1c). $\times$, Phage A1b; 9 , phage Alc.

Fig. 8. Adsorption of phage A 1 b to $S$. typhimurium, strain Q1, with subsequent multiplication. The arrow indicates the point at which phage production begins-approximately 20 min. $\times$, Turbidity curve of $Q 1 ; \square$, turbidity curve of $Q 1+$ phage $A 1 b$;, titre of phage A $1 b$. 
of the others in three experiments out of fifteen. This is in contrast to the other figures set out in this paper, such as the percentage of survivors and the percentage of induced bacteria, where the findings in different experiments have been relatively constant.

Latent period and phage production curve. When an actively growing culture of strain Q1 containing approximately $10^{8}$ bacteria $/ \mathrm{ml}$. was exposed to temperate phage $\mathbf{A} \mathbf{1 b}$ at a concentration calculated to give a phage:bacterium ratio of $1: 1$, the bacteria quickly adsorbed the phage particles. Production of new phage began in about $20 \mathrm{~min}$. and ended in about $90 \mathrm{~min}$. (Fig. 8). Lysogenic strain Q1 (Alb), prepared as for irradiation but incubated without irradiation, showed a steady increase in free phage which reached its maximum in $c .45 \mathrm{~min}$., and thereafter settled down to run at a more or less constant value for the rest of the period of incubation (Fig. 9). Irradiated strain

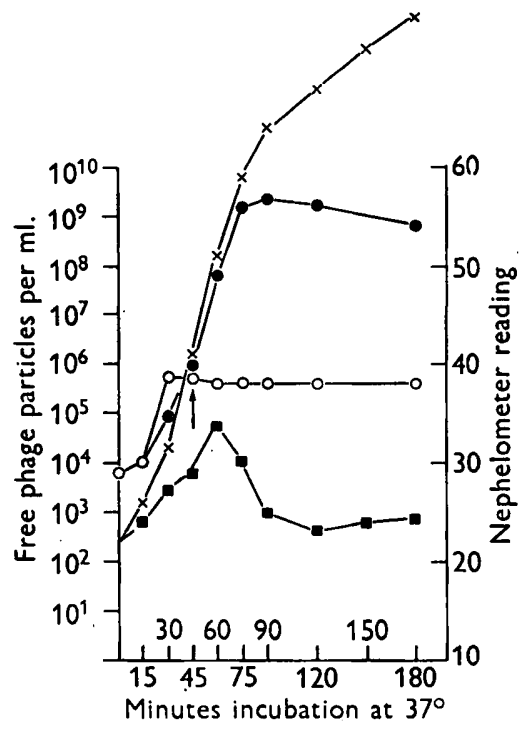

Fig. 9



Fig. 10

Fig. 9. Latent period between irradiation of $S$. typhimurium strain $Q 1(A 1 b)$ and the liberation (as a result of induction) of free phage particles. The arrow (at $45 \mathrm{~min}$.) indicates the point at which the curve of phage production in the irradiated culture breaks away from the curve of 'normal' phage production in the control. $\times$, Turbidity curve of $Q 1$ (AIb); $\square$, turbidity curve of $Q 1$ (AIb) irradiated 5 sec.; $O$, titre of free phage in non-irradiated $Q 1$ (A 1 b); $O$, titre of free phage in irradiated $Q 1$ (A1b).

Fig. 10. Relationship of induction to the degree of damage which $u$.v. radiation produces in the host bacterium. The central column in each section serves as a control, and shows the percentage of bacteria in cultures of $S$. typhimurium, strain Q1 (non-lysogenic with A phage), which survive irradiation. At all degrees of exposure, induction in strain Q1 (A 1 b)-shown in the columns to the left of the control-occurs partly in bacteria which, in terms of the contiol, strain $Q 1$, would have survived irradiation, and partly in bacteria which would not have survived. In strain Q1 (A 1c) (to the right of the control) the bacteria which are induced are mostly those which would not have survived irradiation. As a matter of interest, induction due to phage $B 5 d$ is also shown in this diagram. $\square$, Viable bacteria; 圈, bacteria destroyed by the induction of phage B $5 d$; $\square$, bacteria destroyed by the induction of phage Alb or A lc; $\square$, bacteria destroyed by u.v. irradiation. 
Q1 (A Ib) produced free phage at the same rate as the non-irradiated control for the first $45 \mathrm{~min}$., during which time the curves ran parallel. Thereafter the curves diverged, for the titre of free phage particles in the irradiated culture became rapidly higher between the 45 th and 90 th min., after which it fell slightly as a result of adsorption of the phage particles to the multiplying lysogenic bacteria, which had survived irradiation.

This experiment was carried out with several of the Q1 (A) strains with similar results. Thus, in these lysogenic strains of Salmonella typhimurium, the latent period between irradiation and phage production, when incubation is carried out in a water bath, is $\mathbf{4 5} \mathrm{min}$. This exceeds by $\mathbf{2 5 - 3 0} \mathrm{min}$. the latent period between the exposure of strain Q1 to temperate $A$ phage and the beginning of phage liberation.

\section{DISCUSSION}

All 12 members of the Q1 (A) series of Salmonella typhimurium strains, which differ from each other only in their prophage content, can be induced by u.v. radiation, but the percentage of bacteria in which induction occurs is relatively low. With the technique used it rarely exceeded $40 \%$, and in many cases was much below this figure. The pattern of the reaction in each lysogenic organism is constant and repeatable. The degree of induction cannot be increased beyond a certain point by lengthening the exposure to u.v. radiation. By the methods described, the maximum liberation of free phage particles is achieved by an exposure of from 5 to $10 \mathrm{sec}$. It is noteworthy that $50-75 \%$ of the bacteria in cultures of the parent strain Q1 are killed by such exposures, and many of the survivors are considerably 'damaged'. The low rate of induction in the Q1 (A) series differs from that observed by Lwoff (1953) in Bacillus megaterium where, with the technique Lwoff used, 'almost the entire bacterial population was affected'. The wide variation in burst size is comparable to that observed in other systems (Delbrück, 1945).

The prolonged latent period is similar to that noted in induced Bacillus magaterium by Lwoff et al. (1950) who found that the irradiated bacterium divided twice before productive phage development started. There is, however, no suggestion that the QI (A) series divide after irradiation. This is clearly demonstrated in $Q 1$ (A 1b), where induction occurs mainly in those bacteria which otherwise-in the absence of induction-would have grown, without division, into giant filaments. Nevertheless, as the normal division time of strain $Q 1$, in this particular cultural environment, is about $\mathbf{3 0} \mathrm{min}$. and the normal development time of phage $A 1 b$ is $15-20$ min. (Fig. 8), it seems possible that the liberation of the prophage which ushers in productive development takes place during some disturbance about the time when the nucleus would normally divide.

The main interest of this work centres round the fact that, in certain members of the series, induction is accompanied by clearing of the culture, while in others it is not, a phenomenon well seen in the two strains selected, Q1 (A 1b) and Q1 (A 1c). What causes this difference? The first and most obvious possibility which suggests itself is that clearing may be related to the per- 
centages of bacteria in the culture which are induced. While this must play a part, it is not the only factor involved, for, as can be seen from Table 3, the percentage of bacteria induced in these two types by $2 \frac{1}{2}$ and 5 sec. exposure to $u . v$. radiation is not significantly different, while the percentage of $Q \mathbf{l}$ (A 1c) bacteria induced by $5 \mathrm{sec}$. exposure is higher than the percentage of Q1 (A 1b) exposed for $20 \mathrm{sec}$., yet strain Q1 (A 1b) shows well-marked clearing at all these exposures, while Q1 (A 1c) does not (Figs. 3, 4).

Conceivably, clearing might be due to something in the nature of 'lysisfrom-without' (Andrewes \& Elford, 1932) brought about by the action of a high concentration of phage particles. Before the deoxyribonucleic acid of a phage particle enters the cytoplasm of a bacterium to which it has become adsorbed, the wall of the bacterium is breached by an enzyme present in the tail of the phage (Puck \& Lee, 1954). When a large number of 'holes' of this kind are made at the same time, rupture of the bacterium due to weakening of the cell wall may occur. Lysogenic bacteria do in fact adsorb their homologous phage, but in experiments (unpublished) we have been unable to produce lysis-from-without by exposing lysogenic bacteria to high concentrations of homologous phage. Further, even if such a reaction did occur, there is no reason why it should operate in the case of strain Q1 (A1b) and not in Q1 (A 1c), as adsorption is alike in both strains.

Again, it is possible that 'endolysin' might be liberated from irradiated strain Q1 (A 1b) and not from Q1 (A 1c), but in fact no endolysin could be detected. While the number of viable bacteria decreased during the first 45 min. of incubation of irradiated cultures (Figs. 5, 6) there was no marked difference between the percentage of bacteria killed in strains $Q 1$ (A 1 b) and Q1 (A 1c). Thus there is no evidence to link the clearing in strain Q1 (A 1b) with the action of a lysin.

By taking the percentage of survivors in an irradiated culture of strain Q1 as a control, and comparing with this figure the percentage of survivors and the percentage of 'bursts' in irradiated strains $Q 1$ (A 1b) and Q1 (A 1c), certain significant facts emerge. This comparison is made graphically in Fig. 10. In strain Q1 (A 1b), induction occurs partly in bacteria which would otherwise have survived the effects of irradiation, and partly in bacteria which would not have survived irradiation, more in the former than in the latter. In Q1 (A1c), the majority of the inductions are in bacteria which would not have survived irradiation, so that the remaining viable bacteria are almost as numerous as in the control, Q1.

It might be thought that this destruction of viable bacteria, leaving only a few to multiply and make good those lost by induction, might bring about clearing. Here again a comparison of other experimental findings shows that, whatever part this may play, it is not the sole cause. Strain Q1 (A Ib) when irradiated for $5 \mathrm{sec}$. has a higher percentage of survivors than Q1 (Alc) irradiated for $10 \mathrm{sec}$. (Table 1); the former shows well-marked clearing, the latter shows no clearing (Figs. 3, 4). Nevertheless, the clearing is in part related to the particular bacteria in which induction occurs. Microscopic examination of smears made from incubated irradiated cultures of strains Q1 
and Q1 (A1c) show that these are alike in that a considerable percentage of the bacteria increase in size without dividing for approximately $90 \mathrm{~min}$., becoming up to 6 times their normal size. Similar smears from Q1 (A 1b) reveal only small numbers of enlarged forms, many of which disappear at an early stage, no doubt as the result of induction. From these microscopical findings it may be concluded that induction in strain Q1 (A 1c) occurs in the heavily damaged bacteria in which both growth and division are arrested, whereas in Q1 (A 1b) induction occurs in the more lightly damaged organisms which, in the absence of induction, would have enlarged and ultimately multiplied. This conclusion dovetails neatly into the findings presented in Fig. 10. Thus the clearing in strain Q1 $\left(\begin{array}{ll}A & 1 b\end{array}\right)$ results partly from the induction of a proportion of the non-viable bacteria killed by irradiation, but mainly from the induction of a large proportion of the remaining viable bacteria which disintegrate before they can enlarge. On the other hand, irradiated Q1 (A 1c) fails to clear because the lysis of the non-viable, non-enlarging bacteria is masked by the gross increase in size of the surviving bacteria.

The validity of these conclusions was tested by examining Q1 (A 2d), a strain which when irradiated clears well, though not quite so well as Q1 (A 1b). It was found that induction occurred partly in lightly affected bacteria and partly in organisms which had received a fatal dose of u.v. radiation. Enlarged forms were to be seen in smears, but these were less numerous than in corresponding smears of $Q \mathbf{l}$ (AIC). On the other hand, the percentage of bacteria induced was higher than in Q1 (A 1b) and Q1 (A Ic).

It can therefore be concluded that clearing depends mainly on two factors. The first and most important is the level at which induction takes place in relation to the lethal action of the u.v. radiation on the bacteria. Clearing is most marked when induction occurs in lightly damaged organisms which in the absence of induction would enlarge and ultimately divide. It becomes less obvious where the induced bacteria are those which have received a lethal dose and consequently do not enlarge. The second and less important factor, as far as these particular strains are concerned, is the percentage of bacteria induced, though this must obviously play a part.

The fact that induction occurs at different degrees of irradiation damage is of considerable general interest. The most plausible explanation of induction, and one that commands general acceptance, is that the prophage, normally attached to the chromosome, and in this condition inactive and innocuous, is freed from its attachment by processes initiated by u.v. radiation, and assumes a productive or virulent role. If this theory is valid, it would appear that prophage A Ib is activated by a hit which is less lethal to the host bacterium than the hit or hits which frees prophage A 1c. These findings are in accordance with the conception that the action of u.v. radiation in causing induction is not directly on the prophage, but is on the bacterial host (Jacob, 1954). On the other hand the process is complex, and it is clear that the two prophages, Alb and A 1c, despite their close relationship, have each their own specific triggering mechanism, and are activated by different stimuli, the nature of which has still to be discovered. 


\section{REFERENCES}

ANDrewes, C. H. \& ELFord, W. J. (1932). The 'killing' of bacteria by bacteriophage. Brit. J. exp. Path. 13, 13.

BoYD, J. S. K. (1949). Morphological changes in bacteriophage-infected organisms as revealed by phase-contrast illumination. J. Path. Bact. 61, 127.

Boyı, J. S. K. (1950). The symbiotic bacteriophages of Salmonella typhimurium. J. Path. Bact. 62, 501.

Boyd, J. S. K. \& Bidwell, D. E. (1957). The type A phages of Salmonella typhimurium: identification by a standardized cross-immunity test. J. gen. Microbiol. 16, 217.

Deening, R. A. \& Setrow, R. B. (1957). Inhibition of cell division of Escherichia coli by low doses of ultraviolet light. Science, 126, 397.

Delbrück, M. (1945). The burst size distribution in the growth of bacterial viruses. J. Bact. 50, 131.

Gorrill, R. H. \& GrAy, R. A. (1956). The induction of bacteriophage in staphylococci. J. gen. Microbiol. 14, 167.

$\mathrm{J}_{\mathrm{ACOB}}$, F. (1954). Les bactéries lysogènes et la notion de provirus. Monogr. Inst. Pasteur.

JACOB, F. \& Fuerst, C. R. (1958). The mechanism of lysis by phage studied with defective lysogenic bacteria. J. gen. Microbiol. 18, 518.

Latarjet, R., Morenne, P. \& Berger, R. (1953). Une appareil simple pour le dosage des rayonnements ultraviolets émis par les lampes germicides. Ann. Inst. Pasteur, 85, 174.

LiLLeEngen, K. (1948). Typing of Salmonella typhimurium by means of bacteriophage. Acta path. microbiol. scand. Suppl. 77.

Lwoff, A. (1953). Lysogeny. Bact. Rev. 17, 269.

Lwoff, A., Siminovitch, L. \& KJELdgaArd, N. (1950). Induction de la production des bactériophages chez une bactérie lysogène. Ann. Inst. Pasteur, 79, 815.

PUCK, T. T. \& LEE, H. H. (1954). Mechanism of cell wall penetration by viruses. J. exp. Med. 99, 481.

Visconti, N. \& Delbrück, M. (1953). The mechanism of genetic recombination in phage. Genetics, 38, 5.

Zinder, N. D. \& Lederberg, J. (1952). Genetic exchange in Salmonella. J. Bact. 64, 679 .

(Received 28 May 1959) 